Kragujevac Journal of Mathematics

Volume 42(2) (2018), Pages 217-228.

\title{
THE HARMONIC INDEX OF EDGE-SEMITOTAL GRAPHS, TOTAL GRAPHS AND RELATED SUMS
}

\author{
B. N. ONAGH ${ }^{1}$
}

\begin{abstract}
For a connected graph $G$, there are several related graphs such as line graph $L(G)$, subdivision graph $S(G)$, vertex-semitotal graph $R(G)$, edge-semitotal graph $Q(G)$ and total graph $T(G)$ [I. Gutman, B. Furtula, Ž. K. Vukićević and G. Popivoda, On Zagreb indices and coindices, MATCH Commun. Math. Comput. Chem. 74 (2015), 5-16, W. Yan, B. -Y. Yang and Y. -N. Yeh, The behavior of Wiener indices and polynomials of graphs under five graph decorations, Appl. Math. Lett. 20 (2007), 290-295]. Let $F$ be one of symbols $S, R, Q$ or $T$. The $F$-sum $G_{1}+{ }_{F} G_{2}$ of two connected graphs $G_{1}$ and $G_{2}$ is a graph with vertex set $\left(V\left(G_{1}\right) \cup E\left(G_{1}\right)\right) \times V\left(G_{2}\right)$ in which two vertices $\left(u_{1}, v_{1}\right)$ and $\left(u_{2}, v_{2}\right)$ of $G_{1}+{ }_{F} G_{2}$ are adjacent if and only if $\left[u_{1}=u_{2} \in V\left(G_{1}\right)\right.$ and $\left.v_{1} v_{2} \in E\left(G_{2}\right)\right]$ or $\left[v_{1}=v_{2}\right.$ and $u_{1} u_{2} \in E(F(G))$ ] [M. Eliasi and B. Taeri, Four new sums of graphs and their Wiener indices, Discrete Appl. Math. 157 (2009), 794-803]. In this paper, we investigate the harmonic index of edge-semitotal graphs, total graphs and $F$-sum of graphs, where $F=Q$ or $T$.
\end{abstract}

\section{INTRODUCTION}

Throughout this paper, all graphs are finite, simple, undirected and connected. Let $G$ be a graph with vertex set $V(G)$ and edge set $E(G)$. As usual, the degree of a vertex $u$ in $G$ is denoted by $\operatorname{deg}_{G}(u)$. We will use $P_{n}$ to denote the path of order $n$.

The edge-semitotal graph $Q(G)$ is obtained from $G$ by inserting a new vertex into each edge of $G$, then joining with edges those pairs of new vertices on adjacent edges of $G$ (see Figure 1). The total graph $T(G)$ has as its vertices the edges and vertices of $G$; adjacency in $T(G)$ is defined as adjacency or incidence for the corresponding elements of $G$ (see Figure 1).

Key words and phrases. Harmonic index, line graph, total graph, edge-semitotal graph, $F$-sum. 2010 Mathematics Subject Classification. Primary: 05C07. Secondary: 05C76.

Received: November 24, 2016.

Accepted: February 11, 2017. 

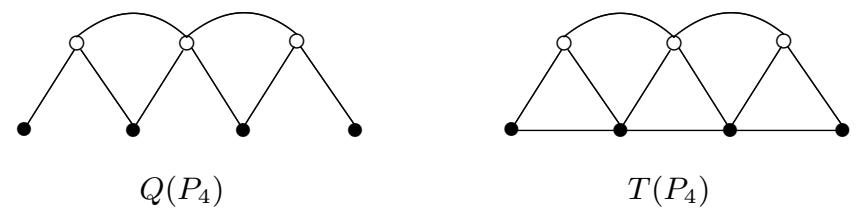

FiguRe 1. $Q\left(P_{4}\right)$ and $T\left(P_{4}\right)$

Let $G_{1}$ and $G_{2}$ be two graphs and $F \in\{Q, T\}$. Then, the $F$-sum $G_{1}+{ }_{F} G_{2}$ has $\left|V\left(G_{2}\right)\right|$ copies of the graph $F\left(G_{1}\right)$ and we can label these copies by vertices of $G_{2}$. The vertices in each copy have two situations: the vertices in $V\left(G_{1}\right)$ (black vertices) and the vertices in $E\left(G_{1}\right)$ (white vertices). Now, we join only black vertices with the same name in $F\left(G_{1}\right)$ in which their corresponding labels are adjacent in $G_{2}$ (see Figure 2).

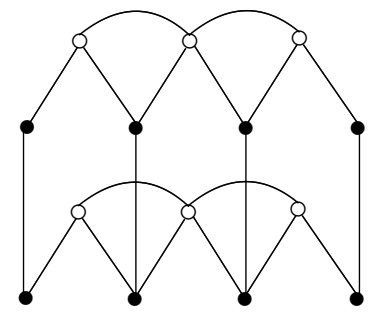

$P_{4}+{ }_{Q} P_{2}$

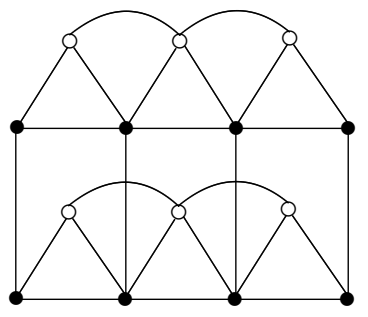

$P_{4}+{ }_{T} P_{2}$

Figure 2. $P_{4}+{ }_{Q} P_{2}$ and $P_{4}+_{T} P_{2}$

The first Zagreb index, the inverse degree and the harmonic index of a graph $G$ are important vertex-degree-based indices related to $G$, where denoted by $M_{1}(G), r(G)$ and $H(G)$, respectively, and defined as follows [3,4]:

$M_{1}(G)=\sum_{u \in V(G)} \operatorname{deg}_{G}^{2}(u), \quad r(G)=\sum_{u \in V(G)} \frac{1}{\operatorname{deg}_{G}(u)}, \quad H(G)=\sum_{u v \in E(G)} \frac{2}{\operatorname{deg}_{G}(u)+\operatorname{deg}_{G}(v)}$.

The first Zagreb index can be expressed as $M_{1}(G)=\sum_{u v \in E(G)}\left(\operatorname{deg}_{G}(u)+\operatorname{deg}_{G}(v)\right)[8]$.

In this paper, we consider the harmonic index. In recent years, this topological index has been extensively studied. Shwetha et al. [11] derived expressions for the harmonic index of the join, corona product, Cartesian product, composition and symmetric difference of graphs. Recently, Onagh investigated the harmonic index of product graphs, subdivision graphs, $t$-subdivision graphs and $F$-sum of graphs, where $F \in\left\{S, S_{t}\right\}[9,10]$. More results on the harmonic index can been found in $[1,6,7,12-15]$.

In this paper, we study the harmonic index of edge-semitotal graphs, total graphs and $F$-sum of graphs, where $F=Q$ or $T$. 


\section{THE HARMONIC INDEX OF EDGE-SEMITOTAL AND TOTAL GRAPHS}

In this section, we give some upper bounds for the harmonic index of edge-semitotal and total graphs. Hereafter, we deal with nontrivial graphs.

Note that $\operatorname{deg}_{Q(G)}(u)=\operatorname{deg}_{G}(u)$ for all $u \in V(G)$ and $\operatorname{deg}_{Q(G)}(w)=\operatorname{deg}_{G}(u)+$ $\operatorname{deg}_{G}(v)=2+\operatorname{deg}_{L(G)}(w)$ for all $w \in V(Q(G))-V(G)$, where $w$ is the vertex inserted into the edge $u v$ of $G$.

Theorem 2.1. Let $G$ be a graph of order $n$ and size $m$. Then

$$
H(Q(G))<\frac{1}{2} H(G)+\frac{1}{4} H(L(G))+\frac{1}{16} M_{1}(G)+\frac{1}{2} n-\frac{1}{8} m .
$$

Proof. By definition of the harmonic index, we have

$$
\begin{aligned}
& H(Q(G)) \\
= & \sum_{u v \in E(G)}\left(\frac{2}{\operatorname{deg}_{Q(G)}(u)+\operatorname{deg}_{Q(G)}(x)}+\frac{2}{\operatorname{deg}_{Q(G)}(x)+\operatorname{deg}_{Q(G)}(v)}\right) \\
& (\text { where } x \text { is the vertex inserted into the edge uv of } G) \\
& +\sum_{w w^{\prime} \in E(L(G))} \frac{2}{\operatorname{leg}_{Q(G)}(w)+\operatorname{deg}_{Q(G)}\left(w^{\prime}\right)} \\
= & \sum_{u v \in E(G)}\left(\frac{2}{\operatorname{deg}_{G}(u)+\left(\operatorname{deg}_{G}(u)+\operatorname{deg}_{G}(v)\right)}+\frac{2}{\left(\operatorname{deg}_{G}(u)+\operatorname{deg}_{G}(v)\right)+\operatorname{deg}_{G}(v)}\right) \\
& +\sum_{w w^{\prime} \in E(L(G))} \frac{2}{\left(2+\operatorname{deg}_{L(G)}(w)\right)+\left(2+\operatorname{deg}_{L(G)}\left(w^{\prime}\right)\right)} \\
= & \sum_{u v \in E(G)}\left(\frac{2}{\operatorname{deg}_{G}(u)+\left(\operatorname{deg}_{G}(u)+\operatorname{deg}_{G}(v)\right)}+\frac{2}{\left(\operatorname{deg}_{G}(u)+\operatorname{deg}_{G}(v)\right)+\operatorname{deg}_{G}(v)}\right) \\
& +\sum_{w w^{\prime} \in E(L(G))} \frac{2}{4+\left(\operatorname{deg}_{L(G)}(w)+\operatorname{deg}_{L(G)}\left(w^{\prime}\right)\right)} \\
:= & \sum_{1}+\sum_{2} 2 .
\end{aligned}
$$

By Jensen's inequality, for every $u v \in E(G)$, we have

$$
\begin{aligned}
\frac{2}{\operatorname{deg}_{G}(u)+\left(\operatorname{deg}_{G}(u)+\operatorname{deg}_{G}(v)\right)} & <\frac{1}{2} \frac{1}{\operatorname{deg}_{G}(u)}+\frac{1}{4} \frac{2}{\operatorname{deg}_{G}(u)+\operatorname{deg}_{G}(v)}, \\
\frac{2}{\left(\operatorname{deg}_{G}(u)+\operatorname{deg}_{G}(v)\right)+\operatorname{deg}_{G}(v)} & <\frac{1}{4} \frac{2}{\operatorname{deg}_{G}(u)+\operatorname{deg}_{G}(v)}+\frac{1}{2} \frac{1}{\operatorname{deg}_{G}(v)} .
\end{aligned}
$$

So,

$$
\sum 1<\frac{1}{2} \sum_{u v \in E(G)}\left(\frac{1}{\operatorname{deg}_{G}(u)}+\frac{1}{\operatorname{deg}_{G}(v)}\right)+\frac{1}{2} \sum_{u v \in E(G)} \frac{2}{\operatorname{deg}_{G}(u)+\operatorname{deg}_{G}(v)}
$$




$$
\begin{aligned}
& =\frac{1}{2} \sum_{u \in V(G)}\left(\frac{1}{\operatorname{deg}_{G}(u)} \times \operatorname{deg}_{G}(u)\right)+\frac{1}{2} H(G) \\
& =\frac{1}{2} n+\frac{1}{2} H(G) .
\end{aligned}
$$

Similarly, for every $w w^{\prime} \in E(L(G))$,

$$
\frac{2}{4+\left(\operatorname{deg}_{L(G)}(w)+\operatorname{deg}_{L(G)}\left(w^{\prime}\right)\right)} \leq \frac{1}{8}+\frac{1}{4} \frac{2}{\operatorname{deg}_{L(G)}(w)+\operatorname{deg}_{L(G)}\left(w^{\prime}\right)},
$$

with equality if and only if $\operatorname{deg}_{L(G)}(w)+\operatorname{deg}_{L(G)}\left(w^{\prime}\right)=4$. This implies that

$$
\begin{aligned}
\sum 2 & \leq \frac{1}{8}|E(L(G))|+\frac{1}{4} H(L(G)) \\
& =\frac{1}{8}\left(\frac{1}{2} M_{1}(G)-m\right)+\frac{1}{4} H(L(G)) \\
& =\frac{1}{16} M_{1}(G)-\frac{1}{8} m+\frac{1}{4} H(L(G)) .
\end{aligned}
$$

Therefore,

$$
H(Q(G))<\frac{1}{2} H(G)+\frac{1}{4} H(L(G))+\frac{1}{16} M_{1}(G)+\frac{1}{2} n-\frac{1}{8} m .
$$

This completes the proof.

Example 2.1. For any $n \geq 4$,

$$
H\left(Q\left(P_{n}\right)\right)=\left(\frac{9}{5}+\frac{2}{3}(n-3)\right)+\left(\frac{4}{7}+\frac{1}{4}(n-4)\right)=\frac{83}{35}+\frac{2}{3}(n-3)+\frac{1}{4}(n-4) .
$$

Note that $\operatorname{deg}_{T(G)}(u)=2 \operatorname{deg}_{G}(u)$ for all $u \in V(G)$ and $\operatorname{deg}_{T(G)}(w)=\operatorname{deg}_{G}(u)+$ $\operatorname{deg}_{G}(v)=2+\operatorname{deg}_{L(G)}(w)$ for all $w \in V(T(G))-V(G)$, where $w$ is the vertex inserted into the edge $u v$ of $G$.

Theorem 2.2. Let $G$ be a graph of order $n$ and size $m$. Then

$$
H(T(G)) \leq H(G)+\frac{1}{4} H(L(G))+\frac{1}{16} M_{1}(G)+\frac{1}{4} n-\frac{1}{8} m,
$$

with equality if and only if $G \cong C_{n}$.

Proof. Note that

$$
\begin{aligned}
& H(T(G)) \\
= & \sum_{u v \in E(G)} \frac{2}{\operatorname{deg}_{T(G)}(u)+\operatorname{deg}_{T(G)}(v)} \\
& +\sum_{u v \in E(G)}\left(\frac{2}{\operatorname{deg}_{T(G)}(u)+\operatorname{deg}_{T(G)}(x)}+\frac{2}{\operatorname{deg}_{T(G)}(x)+\operatorname{deg}_{T(G)}(v)}\right)
\end{aligned}
$$

(where $x$ is the vertex inserted into the edge $u v$ of $G$ ) 


$$
\begin{aligned}
& +\sum_{w w^{\prime} \in E(L(G))} \frac{2}{\operatorname{deg}_{T(G)}(w)+\operatorname{deg}_{T(G)}\left(w^{\prime}\right)} \\
= & \sum_{u v \in E(G)} \frac{2}{2 \operatorname{deg}_{G}(u)+2 \operatorname{deg}_{G}(v)} \\
& +\sum_{u v \in E(G)}\left(\frac{2}{2 \operatorname{deg}_{G}(u)+\left(\operatorname{deg}_{G}(u)+\operatorname{deg}_{G}(v)\right)}\right. \\
& \left.+\frac{2}{\left(\operatorname{deg}_{G}(u)+\operatorname{deg}_{G}(v)\right)+2 \operatorname{deg}_{G}(v)}\right) \\
& +\sum_{w w^{\prime} \in E(L(G))} \frac{2}{\left(2+\operatorname{deg}_{L(G)}(w)\right)+\left(2+\operatorname{deg}_{L(G)}\left(w^{\prime}\right)\right)} \\
= & \frac{1}{2} H(G) \\
& +\sum_{u v \in E(G)}\left(\frac{2}{2 \operatorname{deg}_{G}(u)+\left(\operatorname{deg}_{G}(u)+\operatorname{deg}_{G}(v)\right)}\right. \\
& \left.+\frac{2}{\left(\operatorname{deg}_{G}(u)+\operatorname{deg}_{G}(v)\right)+2 \operatorname{deg}_{G}(v)}\right) \\
& +\sum_{w w^{\prime} \in E(L(G))} \frac{2}{4+\left(\operatorname{deg}_{L(G)}(w)+\operatorname{deg}_{L(G)}\left(w^{\prime}\right)\right)} \\
:= & \frac{1}{2} H(G)+\sum 1+\sum 2 .
\end{aligned}
$$

By Jensen's inequality, for every $u v \in E(G)$, we have

$$
\frac{2}{2 \operatorname{deg}_{G}(u)+\left(\operatorname{deg}_{G}(u)+\operatorname{deg}_{G}(v)\right)} \leq \frac{1}{4} \frac{1}{\operatorname{deg}_{G}(u)}+\frac{1}{4} \frac{2}{\operatorname{deg}_{G}(u)+\operatorname{deg}_{G}(v)},
$$

with equality if and only if $\operatorname{deg}_{G}(u)=\operatorname{deg}_{G}(v)$. Also,

$$
\frac{2}{\left(\operatorname{deg}_{G}(u)+\operatorname{deg}_{G}(v)\right)+2 \operatorname{deg}_{G}(v)} \leq \frac{1}{4} \frac{2}{\operatorname{deg}_{G}(u)+\operatorname{deg}_{G}(v)}+\frac{1}{4} \frac{1}{\operatorname{deg}_{G}(v)},
$$

with equality if and only if $\operatorname{deg}_{G}(u)=\operatorname{deg}_{G}(v)$. Thus,

$$
\begin{aligned}
\sum 1 & \leq \frac{1}{4} \sum_{u v \in E(G)}\left(\frac{1}{\operatorname{deg}_{G}(u)}+\frac{1}{\operatorname{deg}_{G}(v)}\right)+\frac{1}{2} \sum_{u v \in E(G)} \frac{2}{\operatorname{deg}_{G}(u)+\operatorname{deg}_{G}(v)} \\
& =\frac{1}{4} n+\frac{1}{2} H(G) .
\end{aligned}
$$

On the other hand,

$$
\sum 2 \leq \frac{1}{4} H(L(G))+\frac{1}{16} M_{1}(G)-\frac{1}{8} m
$$


with equality if and only if $\operatorname{deg}_{L(G)}(w)+\operatorname{deg}_{L(G)}\left(w^{\prime}\right)=4$ for all $w w^{\prime} \in E(L(G))$. Therefore,

$$
H(T(G)) \leq H(G)+\frac{1}{4} H(L(G))+\frac{1}{16} M_{1}(G)+\frac{1}{4} n-\frac{1}{8} m .
$$

One can see that equality holds in above inequality if and only if the inequalities (2.1), (2.2) and (2.3) be equalities, i.e., $G$ is a $k$-regular such that $\operatorname{deg}_{L(G)}(w)+$ $\operatorname{deg}_{L(G)}\left(w^{\prime}\right)=4(*)$ for all $w w^{\prime} \in L(G)$. Since $L(G)$ is $(2 k-2)$-regular, by $(*)$, we can get $k=2$.

This completes the proof.

Example 2.2. For any $n \geq 4$,

$$
\begin{aligned}
H\left(T\left(P_{n}\right)\right) & =\left(\frac{2}{3}+\frac{1}{4}(n-3)\right)+\left(\frac{48}{35}+\frac{1}{2}(n-3)\right)+\left(\frac{4}{7}+\frac{1}{4}(n-4)\right) \\
& =\frac{274}{105}+\frac{3}{4}(n-3)+\frac{1}{4}(n-4) .
\end{aligned}
$$

\section{The harmonic Index FOR $Q$-SUm And $T$-SUm of GRAPhS}

In the following theorem, we give an upper bound for the harmonic index of $G_{1}+{ }_{Q} G_{2}$ in terms of $H\left(Q\left(G_{1}\right)\right), H\left(L\left(G_{1}\right)\right), H\left(G_{2}\right), M_{1}\left(G_{1}\right), r\left(G_{1}\right)$ and $r\left(G_{2}\right)$.

Theorem 3.1. Let $G_{1}$ and $G_{2}$ be two graphs. Then

$$
\begin{aligned}
H\left(G_{1}+{ }_{Q} G_{2}\right)< & \frac{1}{4} n_{2} H\left(Q\left(G_{1}\right)\right)+\frac{3}{16} n_{2} H\left(L\left(G_{1}\right)\right)+\frac{1}{4} n_{1} H\left(G_{2}\right) \\
& +\frac{3}{64} n_{2} M_{1}(G)+\frac{1}{4} m_{2} r\left(G_{1}\right)+m_{1} r\left(G_{2}\right)-\frac{3}{32} n_{2} m_{1},
\end{aligned}
$$

where $n_{i}=\left|V\left(G_{i}\right)\right|$ and $m_{i}=\left|E\left(G_{i}\right)\right|, i=1,2$.

Proof. Let $\operatorname{deg}(u, v)=\operatorname{deg}_{G_{1}+_{Q} G_{2}}(u, v)$ be the degree of a vertex $(u, v)$ in $G_{1}+_{Q} G_{2}$. Then,

$$
\begin{aligned}
H\left(G_{1}+{ }_{Q} G_{2}\right)= & \sum_{u \in V\left(G_{1}\right)} \sum_{v_{1} v_{2} \in E\left(G_{2}\right)} \frac{2}{\operatorname{deg}\left(u, v_{1}\right)+\operatorname{deg}\left(u, v_{2}\right)} \\
& +\sum_{v \in V\left(G_{2}\right)} \sum_{u_{1} u_{2} \in E\left(Q\left(G_{1}\right)\right)} \frac{2}{\operatorname{deg}\left(u_{1}, v\right)+\operatorname{deg}\left(u_{2}, v\right)} \\
:= & \sum 1+\sum 2 .
\end{aligned}
$$

Note that

$$
\begin{aligned}
\sum 1 & =\sum_{u \in V\left(G_{1}\right)} \sum_{v_{1} v_{2} \in E\left(G_{2}\right)} \frac{2}{\left(\operatorname{deg}_{Q\left(G_{1}\right)}(u)+\operatorname{deg}_{G_{2}}\left(v_{1}\right)\right)+\left(\operatorname{deg}_{Q\left(G_{1}\right)}(u)+\operatorname{deg}_{G_{2}}\left(v_{2}\right)\right)} \\
& =\sum_{u \in V\left(G_{1}\right)} \sum_{v_{1} v_{2} \in E\left(G_{2}\right)} \frac{2}{2 \operatorname{deg}_{Q\left(G_{1}\right)}(u)+\left(\operatorname{deg}_{G_{2}}\left(v_{1}\right)+\operatorname{deg}_{G_{2}}\left(v_{2}\right)\right)}
\end{aligned}
$$




$$
=\sum_{u \in V\left(G_{1}\right)} \sum_{v_{1} v_{2} \in E\left(G_{2}\right)} \frac{2}{2 \operatorname{deg}_{G_{1}}(u)+\left(\operatorname{deg}_{G_{2}}\left(v_{1}\right)+\operatorname{deg}_{G_{2}}\left(v_{2}\right)\right)} .
$$

By Jensen's inequality, for every $u \in V\left(G_{1}\right)$ and every $v_{1} v_{2} \in E\left(G_{2}\right)$, we have (3.1)

$$
\frac{2}{2 \operatorname{deg}_{G_{1}}(u)+\left(\operatorname{deg}_{G_{2}}\left(v_{1}\right)+\operatorname{deg}_{G_{2}}\left(v_{2}\right)\right)} \leq \frac{1}{4} \frac{1}{\operatorname{deg}_{G_{1}}(u)}+\frac{1}{4} \frac{2}{\operatorname{deg}_{G_{2}}\left(v_{1}\right)+\operatorname{deg}_{G_{2}}\left(v_{2}\right)},
$$

with equality if and only if $2 \operatorname{deg}_{G_{1}}(u)=\operatorname{deg}_{G_{2}}\left(v_{1}\right)+\operatorname{deg}_{G_{2}}\left(v_{2}\right)$. Thus,

$$
\begin{aligned}
\sum 1 & \leq \frac{1}{4} \sum_{u \in V\left(G_{1}\right)} \sum_{v_{1} v_{2} \in E\left(G_{2}\right)} \frac{1}{\operatorname{deg}_{G_{1}}(u)}+\frac{1}{4} \sum_{u \in V\left(G_{1}\right)} \sum_{v_{1} v_{2} \in E\left(G_{2}\right)} \frac{2}{\operatorname{deg}_{G_{2}}\left(v_{1}\right)+\operatorname{deg}_{G_{2}}\left(v_{2}\right)} \\
& =\frac{1}{4} \sum_{u \in V\left(G_{1}\right)}\left(m_{2} \times \frac{1}{\operatorname{deg}_{G_{1}}(u)}\right)+\frac{1}{4} \sum_{u \in V\left(G_{1}\right)} H\left(G_{2}\right) \\
& =\frac{1}{4} m_{2} r\left(G_{1}\right)+\frac{1}{4} n_{1} H\left(G_{2}\right) .
\end{aligned}
$$

Also,

$$
\begin{aligned}
& \sum 2=\sum_{v \in V\left(G_{2}\right)} \sum_{\substack{u_{1} u_{2} \in E\left(Q\left(G_{1}\right)\right) \\
u_{1} \in V\left(G_{1}\right), u_{2} \in V\left(Q\left(G_{1}\right)\right)-V\left(G_{1}\right)}} \frac{2}{\operatorname{deg}\left(u_{1}, v\right)+\operatorname{deg}\left(u_{2}, v\right)} \\
& +\sum_{v \in V\left(G_{2}\right)} \sum_{\substack{u_{1} u_{2} \in E\left(Q\left(G_{1}\right)\right) \\
u_{1}, u_{2} \in V\left(Q\left(G_{1}\right)\right)-V\left(G_{1}\right)}} \frac{2}{\operatorname{deg}\left(u_{1}, v\right)+\operatorname{deg}\left(u_{2}, v\right)} \\
& =\sum_{v \in V\left(G_{2}\right)} \sum_{\substack{u_{1} u_{2} \in E\left(Q\left(G_{1}\right)\right) \\
u_{1} \in V\left(G_{1}\right), u_{2} \in V\left(Q\left(G_{1}\right)\right)-V\left(G_{1}\right)}} \frac{2}{\left(\operatorname{deg}_{Q\left(G_{1}\right)}\left(u_{1}\right)+\operatorname{deg}_{G_{2}}(v)\right)+\operatorname{deg}_{Q\left(G_{1}\right)}\left(u_{2}\right)} \\
& +\sum_{v \in V\left(G_{2}\right)} \sum_{u_{1} u_{2} \in E\left(Q\left(G_{1}\right)\right)} \frac{2}{\operatorname{deg}_{Q\left(G_{1}\right)}\left(u_{1}\right)+\operatorname{deg}_{Q\left(G_{1}\right)}\left(u_{2}\right)} . \\
& u_{1}, u_{2} \in V\left(Q\left(G_{1}\right)\right)-V\left(G_{1}\right)
\end{aligned}
$$

For every $v \in V\left(G_{2}\right)$ and every $u_{1} u_{2} \in E\left(Q\left(G_{1}\right)\right)$ with $u_{1} \in V\left(G_{1}\right)$ and $u_{2} \in$ $V\left(Q\left(G_{1}\right)\right)-V\left(G_{1}\right)$, we have

$$
\begin{aligned}
\frac{2}{\left(\operatorname{deg}_{Q\left(G_{1}\right)}\left(u_{1}\right)+\operatorname{deg}_{Q\left(G_{1}\right)}\left(u_{2}\right)\right)+\operatorname{deg}_{G_{2}}(v)} \leq & \frac{1}{4} \frac{2}{\operatorname{deg}_{Q\left(G_{1}\right)}\left(u_{1}\right)+\operatorname{deg}_{Q\left(G_{1}\right)}\left(u_{2}\right)} \\
& +\frac{1}{2} \frac{1}{\operatorname{deg}_{G_{2}}(v)},
\end{aligned}
$$

with equality if and only if $\operatorname{deg}_{Q\left(G_{1}\right)}\left(u_{1}\right)+\operatorname{deg}_{Q\left(G_{1}\right)}\left(u_{2}\right)=\operatorname{deg}_{G_{2}}(v)$. Thus,

$$
\sum_{\substack{u_{1} u_{2} \in E\left(Q\left(G_{1}\right)\right) \\ u_{1} \in V\left(G_{1}\right), u_{2} \in V\left(Q\left(G_{1}\right)\right)-V\left(G_{1}\right)}} \frac{2}{\left(\operatorname{deg}_{Q\left(G_{1}\right)}\left(u_{1}\right)+\operatorname{deg}_{G_{2}}(v)\right)+\operatorname{deg}_{Q\left(G_{1}\right)}\left(u_{2}\right)}
$$




$$
\begin{aligned}
\leq & \frac{1}{4} \sum_{\substack{u_{1} u_{2} \in E\left(Q\left(G_{1}\right)\right) \\
u_{1} \in V\left(G_{1}\right), u_{2} \in V\left(Q\left(G_{1}\right)\right)-V\left(G_{1}\right)}} \frac{2}{\operatorname{deg}_{Q\left(G_{1}\right)}\left(u_{1}\right)+\operatorname{deg}_{Q\left(G_{1}\right)}\left(u_{2}\right)} \\
& +\frac{1}{2} \sum_{\substack{u_{1} u_{2} \in E\left(Q\left(G_{1}\right)\right) \\
u_{1} \in V\left(G_{1}\right), u_{2} \in V\left(Q\left(G_{1}\right)\right)-V\left(G_{1}\right)}} \frac{1}{\operatorname{deg}_{G_{2}}(v)} \\
= & \frac{1}{4} \sum_{u_{1} u_{1}^{\prime} \in E\left(G_{1}\right)}\left(\frac{2}{\operatorname{leg}_{Q\left(G_{1}\right)}\left(u_{1}\right)+\operatorname{deg}_{Q\left(G_{1}\right)}\left(u_{2}\right)}+\frac{2}{\operatorname{leg}_{Q\left(G_{1}\right)}\left(u_{2}\right)+\operatorname{deg}_{Q\left(G_{1}\right)}\left(u_{1}^{\prime}\right)}\right)
\end{aligned}
$$

(where $u_{2}$ is the vertex inserted into the edge $u_{1} u_{1}^{\prime}$ of $G_{1}$ )

$$
\begin{aligned}
& +\frac{m_{1}}{\operatorname{deg}_{G_{2}}(v)} \\
= & \frac{1}{4}\left(H\left(Q\left(G_{1}\right)\right)-\sum_{w w^{\prime} \in E\left(L\left(G_{1}\right)\right)} \frac{2}{\operatorname{leg}_{Q\left(G_{1}\right)}(w)+\operatorname{deg}_{Q\left(G_{1}\right)}\left(w^{\prime}\right)}\right)+\frac{m_{1}}{\operatorname{deg}_{G_{2}}(v)} .
\end{aligned}
$$

So,

$$
\begin{aligned}
\sum 2 \leq & \frac{1}{4} \sum_{v \in V\left(G_{2}\right)}\left(H\left(Q\left(G_{1}\right)\right)-\sum_{w w^{\prime} \in E\left(L\left(G_{1}\right)\right)} \frac{2}{\operatorname{deg}_{Q\left(G_{1}\right)}(w)+\operatorname{deg}_{Q\left(G_{1}\right)}\left(w^{\prime}\right)}\right) \\
& +\sum_{v \in V\left(G_{2}\right)} \frac{m_{1}}{\operatorname{deg}_{G_{2}}(v)} \\
& +\sum_{v \in V\left(G_{2}\right)} \sum_{u_{1} u_{2} \in E\left(Q\left(G_{1}\right)\right)} \frac{2}{\operatorname{deg}_{Q\left(G_{1}\right)}\left(u_{1}\right)+\operatorname{deg}_{Q\left(G_{1}\right)}\left(u_{2}\right)} \\
= & \left.\frac{1}{4} n_{2} H\left(Q\left(G_{1}\right)\right)+m_{1} r\left(G_{1}\right)\right)-V\left(G_{1}\right) \\
& -\frac{1}{4} n_{2} \sum_{w w^{\prime} \in E\left(L\left(G_{1}\right)\right)} \frac{2}{\operatorname{deg}_{Q\left(G_{1}\right)}(w)+\operatorname{deg}_{Q\left(G_{1}\right)}\left(w^{\prime}\right)} \\
& +n_{2} \sum_{u_{1} u_{2} \in E\left(L\left(G_{1}\right)\right)} \frac{2}{\operatorname{deg}_{Q\left(G_{1}\right)}\left(u_{1}\right)+\operatorname{deg}_{Q\left(G_{1}\right)}\left(u_{2}\right)} \\
= & \frac{1}{4} n_{2} H\left(Q\left(G_{1}\right)\right)+m_{1} r\left(G_{2}\right)+\frac{3}{4} n_{2} \sum_{w w^{\prime} \in E\left(L\left(G_{1}\right)\right)} \overline{\operatorname{deg}_{Q\left(G_{1}\right)}(w)+\operatorname{deg}_{Q\left(G_{1}\right)}\left(w^{\prime}\right)} \\
= & \frac{1}{4} n_{2} H\left(Q\left(G_{1}\right)\right)+m_{1} r\left(G_{2}\right) \\
& +\frac{3}{4} n_{2} \sum_{w w^{\prime} \in E\left(L\left(G_{1}\right)\right)} \frac{2}{4+\left(\operatorname{deg}_{L\left(G_{1}\right)}(w)+\operatorname{deg}_{L\left(G_{1}\right)}\left(w^{\prime}\right)\right)} .
\end{aligned}
$$


On the other hand,

$$
\sum_{w w^{\prime} \in E\left(L\left(G_{1}\right)\right)} \frac{2}{4+\left(\operatorname{deg}_{L\left(G_{1}\right)}(w)+\operatorname{deg}_{L\left(G_{1}\right)}\left(w^{\prime}\right)\right)} \leq \frac{1}{4} H\left(L\left(G_{1}\right)\right)+\frac{1}{16} M_{1}\left(G_{1}\right)-\frac{1}{8} m_{1},
$$

with equality if and only if $\operatorname{deg}_{L\left(G_{1}\right)}(w)+\operatorname{deg}_{L\left(G_{1}\right)}\left(w^{\prime}\right)=4$ for all $w w^{\prime} \in E\left(L\left(G_{1}\right)\right)$. Therefore,

$$
\begin{aligned}
H\left(G_{1}+{ }_{Q} G_{2}\right) \leq & \frac{1}{4} n_{2} H\left(Q\left(G_{1}\right)\right)+\frac{3}{16} n_{2} H\left(L\left(G_{1}\right)\right)+\frac{1}{4} n_{1} H\left(G_{2}\right) \\
& +\frac{3}{64} n_{2} M_{1}(G)+\frac{1}{4} m_{2} r\left(G_{1}\right)+m_{1} r\left(G_{2}\right)-\frac{3}{32} n_{2} m_{1} .
\end{aligned}
$$

Now, suppose that there exist two graphs $G_{1}$ and $G_{2}$ such that equality holds in above inequality. Then, the inequalities (3.1), (3.2) and (3.3) must be equalities. So, $G_{1}$ and $G_{2}$ are $k_{1}$-regular and $k_{2}$-regular graphs, respectively, such that $2 k_{1}=k_{2}+k_{2}$, $k_{1}+\left(k_{1}+k_{1}\right)=k_{2}$ and $4=\left(2 k_{1}-2\right)+\left(2 k_{1}-2\right)$, a contradiction.

This completes the proof.

Example 3.1. For any $n \geq 4$,

$$
\begin{aligned}
H\left(P_{n}+{ }_{Q} P_{2}\right) & =\left(1+\frac{1}{3}(n-2)\right)+2\left(\frac{22}{15}+\frac{4}{7}(n-3)+\frac{4}{7}+\frac{1}{4}(n-4)\right) \\
& =\frac{533}{105}+\frac{1}{3}(n-2)+\frac{8}{7}(n-3)+\frac{1}{2}(n-4) .
\end{aligned}
$$

Now, we obtain an upper bound for the harmonic index of $G_{1}+{ }_{T} G_{2}$ in terms of $H\left(T\left(G_{1}\right)\right), H\left(L\left(G_{1}\right)\right), H\left(G_{2}\right), M_{1}\left(G_{1}\right), r\left(G_{1}\right)$ and $r\left(G_{2}\right)$.

Theorem 3.2. Let $G_{1}$ and $G_{2}$ be two graphs. Then

$$
\begin{aligned}
H\left(G_{1}+_{T} G_{2}\right)< & \frac{1}{4} n_{2} H\left(T\left(G_{1}\right)\right)+\frac{3}{16} n_{2} H\left(L\left(G_{1}\right)\right)+\frac{1}{4} n_{1} H\left(G_{2}\right) \\
& +\frac{3}{64} n_{2} M_{1}(G)+\frac{1}{8} m_{2} r\left(G_{1}\right)+\frac{5}{4} m_{1} r\left(G_{2}\right)-\frac{3}{32} n_{2} m_{1},
\end{aligned}
$$

where $n_{i}=\left|V\left(G_{i}\right)\right|$ and $m_{i}=\left|E\left(G_{i}\right)\right|, i=1,2$.

Proof. Let $\operatorname{deg}(u, v)=\operatorname{deg}_{G_{1} T_{T} G_{2}}(u, v)$ be the degree of a vertex $(u, v)$ in $G_{1}+_{T} G_{2}$. By definition of harmonic index, we have

$$
\begin{aligned}
H\left(G_{1}+{ }_{T} G_{2}\right)= & \sum_{u \in V\left(G_{1}\right)} \sum_{v_{1} v_{2} \in E\left(G_{2}\right)} \frac{2}{\operatorname{deg}\left(u, v_{1}\right)+\operatorname{deg}\left(u, v_{2}\right)} \\
& +\sum_{v \in V\left(G_{2}\right)} \sum_{u_{1} u_{2} \in E\left(T\left(G_{1}\right)\right)} \frac{2}{\operatorname{deg}\left(u_{1}, v\right)+\operatorname{deg}\left(u_{2}, v\right)} \\
:= & \sum 1+\sum 2 .
\end{aligned}
$$


Note that

$$
\begin{aligned}
\sum 1 & =\sum_{u \in V\left(G_{1}\right)} \sum_{v_{1} v_{2} \in E\left(G_{2}\right)} \frac{2}{\left(\operatorname{deg}_{T\left(G_{1}\right)}(u)+\operatorname{deg}_{G_{2}}\left(v_{1}\right)\right)+\left(\operatorname{deg}_{T\left(G_{1}\right)}(u)+\operatorname{deg}_{G_{2}}\left(v_{2}\right)\right)} \\
& =\sum_{u \in V\left(G_{1}\right)} \sum_{v_{1} v_{2} \in E\left(G_{2}\right)} \frac{2}{2 \operatorname{deg}_{T\left(G_{1}\right)}(u)+\left(\operatorname{deg}_{G_{2}}\left(v_{1}\right)+\operatorname{deg}_{G_{2}}\left(v_{2}\right)\right)} \\
& =\sum_{u \in V\left(G_{1}\right)} \sum_{v_{1} v_{2} \in E\left(G_{2}\right)} \frac{2}{4 \operatorname{deg}_{G_{1}}(u)+\left(\operatorname{deg}_{G_{2}}\left(v_{1}\right)+\operatorname{deg}_{G_{2}}\left(v_{2}\right)\right)} .
\end{aligned}
$$

By similar argument as in the proof of Theorem 3.1, one can show that

$$
\sum 1 \leq \frac{1}{8} m_{2} r\left(G_{1}\right)+\frac{1}{4} n_{1} H\left(G_{2}\right),
$$

with equality if and only if $4 \operatorname{deg}_{G_{1}}(u)=\operatorname{deg}_{G_{2}}\left(v_{1}\right)+\operatorname{deg}_{G_{2}}\left(v_{2}\right)$ for all $u \in V\left(G_{1}\right)$ and all $v_{1} v_{2} \in E\left(G_{2}\right)$.

On the other hand,

$$
\begin{aligned}
& \sum 2=\sum_{v \in V\left(G_{2}\right)} \sum_{u_{1} u_{2} \in E\left(T\left(G_{1}\right)\right)} \frac{2}{\operatorname{deg}\left(u_{1}, v\right)+\operatorname{deg}\left(u_{2}, v\right)} \\
& u_{1}, u_{2} \in V\left(G_{1}\right) \\
& +\sum_{v \in V\left(G_{2}\right)} \sum_{u_{1} u_{2} \in E\left(T\left(G_{1}\right)\right)} \frac{2}{\operatorname{deg}\left(u_{1}, v\right)+\operatorname{deg}\left(u_{2}, v\right)} \\
& u_{1} \in V\left(G_{1}\right), u_{2} \in V\left(T\left(G_{1}\right)\right)-V\left(G_{1}\right) \\
& +\sum_{v \in V\left(G_{2}\right)} \sum_{\substack{u_{1} u_{2} \in E\left(T\left(G_{1}\right)\right) \\
u_{1}, u_{2} \in V\left(T\left(G_{1}\right)\right)-V\left(G_{1}\right)}} \frac{2}{\operatorname{deg}\left(u_{1}, v\right)+\operatorname{deg}\left(u_{2}, v\right)} \\
& =\sum_{v \in V\left(G_{2}\right)} \sum_{u_{1} u_{2} \in E\left(T\left(G_{1}\right)\right)} \frac{2}{\left(\operatorname{deg}_{T\left(G_{1}\right)}\left(u_{1}\right)+\operatorname{deg}_{G_{2}}(v)\right)+\left(\operatorname{deg}_{T\left(G_{1}\right)}\left(u_{2}\right)+\operatorname{deg}_{G_{2}}(v)\right)} \\
& +\sum_{v \in V\left(G_{2}\right)} \sum_{u_{1} u_{2} \in E\left(T\left(G_{1}\right)\right)} \frac{2}{\left(\operatorname{deg}_{T\left(G_{1}\right)}\left(u_{1}\right)+\operatorname{deg}_{G_{2}}(v)\right)+\operatorname{deg}_{T\left(G_{1}\right)}\left(u_{2}\right)} \\
& u_{1} \in V\left(G_{1}\right), u_{2} \in V\left(T\left(G_{1}\right)\right)-V\left(G_{1}\right) \\
& +\sum_{v \in V\left(G_{2}\right)} \sum_{\substack{u_{1} u_{2} \in E\left(T\left(G_{1}\right)\right) \\
u_{1}, u_{2} \in V\left(T\left(G_{1}\right)\right)-V\left(G_{1}\right)}} \frac{2}{\operatorname{deg}_{T\left(G_{1}\right)}\left(u_{1}\right)+\operatorname{deg}_{T\left(G_{1}\right)}\left(u_{2}\right)} \\
& =\sum_{v \in V\left(G_{2}\right)} \sum_{\substack{u_{1} u_{2} \in E\left(T\left(G_{1}\right)\right) \\
u_{1}, u_{2} \in V\left(G_{1}\right)}} \frac{2}{\left(\operatorname{deg}_{T\left(G_{1}\right)}\left(u_{1}\right)+\operatorname{deg}_{T\left(G_{1}\right)}\left(u_{2}\right)\right)+2 \operatorname{deg}_{G_{2}}(v)} \\
& +\sum_{v \in V\left(G_{2}\right)} \sum_{u_{1} u_{2} \in E\left(T\left(G_{1}\right)\right)} \frac{2}{\left(\operatorname{deg}_{T\left(G_{1}\right)}\left(u_{1}\right)+\operatorname{deg}_{T\left(G_{1}\right)}\left(u_{2}\right)\right)+\operatorname{deg}_{G_{2}}(v)}
\end{aligned}
$$
$u_{1} \in V\left(G_{1}\right), u_{2} \in V\left(T\left(G_{1}\right)\right)-V\left(G_{1}\right)$ 


$$
\begin{aligned}
& +\sum_{v \in V\left(G_{2}\right)} \sum_{u_{1} u_{2} \in E\left(L\left(G_{1}\right)\right)} \frac{2}{\operatorname{deg}_{T\left(G_{1}\right)}\left(u_{1}\right)+\operatorname{deg}_{T\left(G_{1}\right)}\left(u_{2}\right)} \\
= & \sum_{v \in V\left(G_{2}\right)} \sum_{u_{1} u_{2} \in E\left(G_{1}\right)} \frac{2}{\left(2 \operatorname{deg}_{G_{1}}\left(u_{1}\right)+2 \operatorname{deg}_{G_{1}}\left(u_{2}\right)\right)+2 \operatorname{deg}_{G_{2}}(v)} \\
& +\sum_{v \in V\left(G_{2}\right)} \sum_{\substack{u_{1} u_{2} \in E\left(T\left(G_{1}\right)\right) \\
u_{1} \in V\left(G_{1}\right), u_{2} \in V\left(T\left(G_{1}\right)\right)-V\left(G_{1}\right)}} \frac{2}{\left(\operatorname{deg}_{T\left(G_{1}\right)}\left(u_{1}\right)+\operatorname{deg}_{T\left(G_{1}\right)}\left(u_{2}\right)\right)+\operatorname{deg}_{G_{2}}(v)} \\
& +\sum_{v \in V\left(G_{2}\right)} \sum_{u_{1} u_{2} \in E\left(L\left(G_{1}\right)\right)} \frac{2}{\operatorname{deg}_{T\left(G_{1}\right)}\left(u_{1}\right)+\operatorname{deg}_{T\left(G_{1}\right)}\left(u_{2}\right)} .
\end{aligned}
$$

One can prove that

$$
\begin{aligned}
\sum 2 \leq & \frac{1}{8} n_{2} H\left(G_{1}\right)+\frac{1}{4} m_{1} r\left(G_{2}\right) \\
& +\frac{1}{4} n_{2} H\left(T\left(G_{1}\right)\right)-\frac{1}{8} n_{2} H\left(G_{1}\right)+m_{1} r\left(G_{2}\right) \\
& +\frac{3}{4} n_{2}\left(\frac{1}{4} H\left(L\left(G_{1}\right)\right)+\frac{1}{16} M_{1}\left(G_{1}\right)-\frac{1}{8} m_{1}\right) .
\end{aligned}
$$

Equality holds in above inequality if and only if

$$
\operatorname{deg}_{G_{1}}\left(u_{1}\right)+\operatorname{deg}_{G_{1}}\left(u_{2}\right)=\operatorname{deg}_{G_{2}}(v)
$$

for all $v \in V\left(G_{2}\right)$ and all $u_{1} u_{2} \in E\left(G_{1}\right)$,

$$
\operatorname{deg}_{T\left(G_{1}\right)}\left(u_{1}\right)+\operatorname{deg}_{T\left(G_{1}\right)}\left(u_{2}\right)=\operatorname{deg}_{G_{2}}(v)
$$

for all $v \in V\left(G_{2}\right)$ and all $u_{1} u_{2} \in E\left(T\left(G_{1}\right)\right)$ with $u_{1} \in V\left(G_{1}\right)$ and $u_{2} \in V\left(T\left(G_{1}\right)\right)-$ $V\left(G_{1}\right)$, and

$$
\operatorname{deg}_{L\left(G_{1}\right)}(w)+\operatorname{deg}_{L\left(G_{1}\right)}\left(w^{\prime}\right)=4,
$$

for all $w w^{\prime} \in E\left(L\left(G_{1}\right)\right)$. Therefore,

$$
\begin{aligned}
H\left(G_{1}+{ }_{T} G_{2}\right) \leq & \frac{1}{4} n_{2} H\left(T\left(G_{1}\right)\right)+\frac{3}{16} n_{2} H\left(L\left(G_{1}\right)\right)+\frac{1}{4} n_{1} H\left(G_{2}\right) \\
& +\frac{3}{64} n_{2} M_{1}(G)+\frac{1}{8} m_{2} r\left(G_{1}\right)+\frac{5}{4} m_{1} r\left(G_{2}\right)-\frac{3}{32} n_{2} m_{1} .
\end{aligned}
$$

Moreover, equality holds in (3.8) if and only the inequalities (3.4), (3.5), (3.6) and (3.7) be equalities, i.e., $G_{1}$ and $G_{2}$ are $k_{1}$-regular and $k_{2}$-regular graphs, respectively, such that $4 k_{1}=k_{2}+k_{2}, k_{1}+k_{1}=k_{2}, 2 k_{1}+\left(k_{1}+k_{1}\right)=k_{2}$ and $\left(2 k_{1}-2\right)+\left(2 k_{1}-2\right)=4$, a contradiction. This completes the proof.

Example 3.2. For any $n \geq 4$,

$$
\begin{aligned}
H\left(P_{n}+_{T} P_{2}\right) & =\left(\frac{2}{3}+\frac{1}{5}(n-2)\right)+2\left(\frac{1}{2}+\frac{1}{5}(n-3)+\frac{7}{6}+\frac{4}{9}(n-3)+\frac{4}{7}+\frac{1}{4}(n-4)\right) \\
& =\frac{36}{7}+\frac{1}{5}(n-2)+\frac{58}{45}(n-3)+\frac{1}{2}(n-4) .
\end{aligned}
$$




\section{REFERENCES}

[1] H. Deng, S. Balachandran, S. K. Ayyaswamy and Y. B. Venkatakrishnan, On the harmonic index and the chromatic number of a graph, Discrete Appl. Math. 161 (2013), 2740-2744.

[2] M. Eliasi and B. Taeri, Four new sums of graphs and their Wiener indices, Discrete Appl. Math. 157 (2009), 794-803.

[3] S. Fajtlowicz, On conjectures of graffiti II, Congr. Numer. 60 (1987), 189-197.

[4] I. Gutman and N. Trinajstić, Graph theory and molecular orbitals. Total $\pi$-electron energy of alternant hydrocarbons, Chem. Phys. Lett. 17 (1972), 535-538.

[5] I. Gutman, B. Furtula, Ž. K. Vukićević and G. Popivoda, On Zagreb indices and coindices, MATCH Commun. Math. Comput. Chem. 74 (2015), 5-16.

[6] J. -B. Lv and J. Li, On the harmonic index and the matching numbers of trees, Ars Combin. 116 (2014), 407-416.

[7] J. -B. Lv, J. Li and W. C. Shiu, The harmonic index of unicyclic graphs with given matching number, Kragujevac J. Math. 38(1) (2014), 173-182.

[8] S. Nikolić, G. Kovačević, A. Miličević and N. Trinajstić, The Zagreb indices 30 years after, Croat. Chem. Acta 76(2) (2003), 113-124.

[9] B. N. Onagh, The harmonic index of subdivision graphs, Trans. Comb. 6(4) (2017), 15-27.

[10] B. N. Onagh, The harmonic index of product graphs, Math. Sci. 11(3) (2017), 203-209.

[11] B. S. Shwetha, V. Lokesha and P. S. Ranjini, On the harmonic index of graph operations, Trans. Comb. 4(4) (2015), 5-14.

[12] L. Zhong, The harmonic index for graphs, Appl. Math. Lett. 25 (2012), 561-566.

[13] L. Zhong, The harmonic index on unicyclic graphs, Ars Combin. 104 (2012), 261-269.

[14] L. Zhong, On the harmonic index and the girth for graphs, Rom. J. Inf. Sci. Tech. 16(4) (2013), 253-260.

[15] L. Zhong and K. Xu, The harmonic index for bicyclic graphs, Util. Math. 90 (2013), 23-32.

[16] W. Yan, B. -Y. Yang and Y. -N. Yeh, The behavior of Wiener indices and polynomials of graphs under five graph decorations, Appl. Math. Lett. 20 (2007), 290-295.

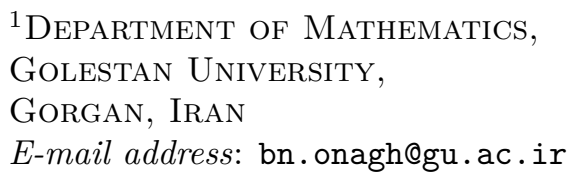

